\title{
Fermi bubble simulations: black hole feedback in the Milky Way
}

\author{
M. Ruszkowski ${ }^{1}$, H.-Y. K. Yang ${ }^{1}$ and E. Zweibel ${ }^{2}$ \\ ${ }^{1}$ Department of Astronomy, University of Michigan, Ann Arbor, MI, USA \\ email: mateuszr@umich.edu, hsyang@umich.edu \\ ${ }^{2}$ Department of Astronomy and Physics, University of Wisconsin Madison, Madison, WI, USA \\ email: zweibel@astro.wisc.edu
}

\begin{abstract}
The Fermi $\gamma$-ray telescope discovered a pair of bubbles at the Galactic center. These structures are spatially-correlated with the microwave emission detected by the WMAP and Planck satellites. These bubbles were likely inflated by a jet launched from the vicinity of a supermassive black hole in the Galactic center. Using MHD simulations, which self-consistently include interactions between cosmic rays and magnetic fields, we build models of the supersonic jet propagation, cosmic ray transport, and the magnetic field amplification within the Fermi bubbles. Our key findings are that: (1) the synthetic Fermi $\gamma$-ray and WMAP microwave spectra based on our simulations are consistent with the observations, suggesting that a single population of cosmic ray leptons may simultaneously explain the emission across a range of photon energies; (2) the model fits the observed centrally-peaked microwave emission if a second, more recent, pair of jets embedded in the Fermi bubbles is included in the model. This is consistent with the observationally-based suggestion made by Su \& Finkbeiner (2012); (3) the radio emission from the bubbles is expected to be strongly polarized due to the relatively high level of field ordering caused by elongated turbulent vortices. This effect is caused by the interaction of the shocks driven by the jets with the preexisting interstellar medium turbulence; (4) a layer of enhanced rotation measure in the shock-compressed region could exist in the bubble vicinity but the level of this enhancement depends on the details of the magnetic topology.
\end{abstract}

Keywords. AGN feedback, cosmic rays, Fermi bubbles, Galactic center

\section{Introduction}

The Fermi bubbles are large-scale bipolar outflow bubbles in the Galaxy that were recently detected with the Fermi observatory ( $\mathrm{Su}$ et al. 2010). They are a unique example of supermassive black hole feedback in our own "backyard" rather than in a distant extragalactic source (for alternative interpretation see, e.g., Crocker \& Aharonian (2011); contributions by Lacki and Dogiel in these proceedings). The Fermi bubbles have received a lot of attention in the literature since their discovery and are one of the most exciting findings from the NASA's Fermi mission. The bubbles are characterized by a flat $\gamma$ ray intensity distribution, hard spectrum extending up to $\sim 100 \mathrm{GeV}$, and sharp $\gamma$-ray edges separating the bubbles and ambient interstellar medium. The bubbles are also visible in the WMAP and Planck data. This emission is referred to as the 'WMAP haze' (Finkbeiner 2004) or Planck haze (Planck Collaboration 2013). This microwave haze emission is centrally-peaked, i.e., its intensity is strongest near the Galactic center.

\section{MHD simulations of the Fermi bubbles}

Using 3D MHD simulations that include cosmic ray physics, we predict observational signatures of the Fermi bubbles in the leptonic AGN jet scenario (Yang et al. 2013, Yang 

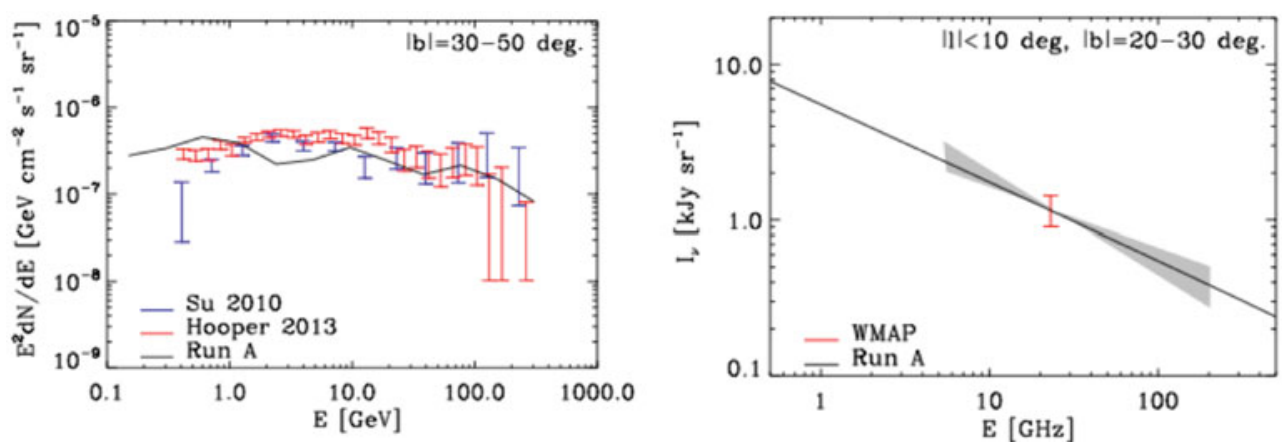

Figure 1. $\Gamma$-ray (left) and microwave (right) spectra of the Fermi bubbles. Solid lines represent model predictions. $l$ and $b$ denote Galactic longitude and latitude, respectively. [A COLOR VERSion is AVAILAbLE ONLINE.]

et al. 2012). In this model, the $\gamma$-ray emission is due to the inverse Compton scattering of the cosmic microwave background, and the interstellar radiation field, by the cosmic ray electrons inside the jet-inflated bubbles, while the WMAP haze emission is due to the synchrotron emission produced by the same population of cosmic rays interacting with the magnetic fields inside the bubbles.

One important aspect of the model is that the cosmic rays tend to accumulate close to the bubble surface. That is, the intrinsic spatial distribution of the cosmic rays is edge-brightened. This naturally leads to a flat distribution of the sky-projected $\gamma$-ray emission of the bubbles, which is in agreement with observations. An appealing feature of this model is that the inflation of the bubbles is so rapid that the age of the bubbles is shorter than the cooling time of cosmic ray electrons. This ensures that the cosmic ray cooling losses do not significantly alter the spectrum, which remains hard up to energies as high as $\sim 100 \mathrm{GeV}$ as observed (see Figure 1).

If we assume an observationally motivated distribution of the ambient Galactic magnetic fields (Strong et al. 2007), we can combine it with the simulated cosmic ray distribution to match the observed slope and normalization of the WMAP synchrotron emission (see Figure 1). We stress that this match between the simulated and observed $\gamma$-ray and microwave spectra can be obtained by invoking a single population of cosmic ray leptons. Thus, the model combined with observations allows us to essentially look inside the Fermi bubbles. Interestingly, in the leptonic model, the cosmic rays contribute relatively little pressure compared to the pressure of thermal gas inside the bubbles. Finkbeiner (these proceedings) reported on a cutoff in the $\gamma$-ray spectrum beyond $\sim 100 \mathrm{GeV}$. This observation will help to put additional constraints on the cosmic ray energy spectra, cooling/heating mechanisms, and the bubble dynamics early in its evolution.

Magnetic field amplification inside the Fermi bubbles As the bubbles expand, the ambient ISM magnetic fields drape around the bubbles. Assuming cosmic ray diffusion is anisotropic (Yang et al. 2012), this prevents the cosmic rays from readily diffusing out of the bubbles, and thus accounts for their sharpness in the $\gamma$-ray emission. While the draping mechanism helps to explain this aspect of the observations, it reduces the strength of magnetic fields inside the bubbles. That is, the bubbles are effectively sealed off from the ambient magnetized ISM and the magnetic fields inside them are reduced due to adiabatic expansion. Consequently, the cosmic rays inside the bubbles are in contact with relatively weak magnetic fields and the predicted microwave emission is too weak compared to observations. 

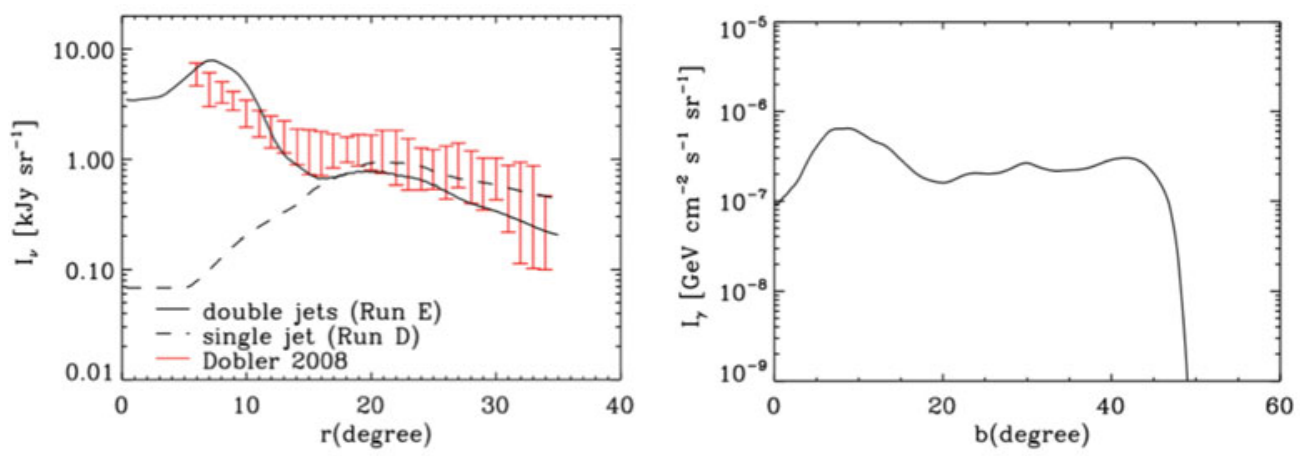

Figure 2. Microwave (left) and $\gamma$-ray (right) intensity profiles.

[A COLOR VERSION IS AVAILABLE ONLINE.]

We suggest that one solution to this problem is to allow for partial draping of the ambient field. In order to study this possibility, we consider tangled ambient magnetic fields characterized by a range of coherence lengths. Our fiducial model consist of the global tangled field superimposed on a more tangled field component closer to the disk plane. We find that decreasing the coherence length of the latter B-field component does lead to partial magnetic draping of the bubbles while not violating the observational constraints on the sharpness of the bubble-ISM interface seen in the $\gamma$-ray emission. At the same time, the reduced coherence length of the ambient field allows for some mixing-in of the ambient field into the bubbles. We demonstrated that, as this coherence length is reduced, the field strength inside the bubbles asymptotically tends to a value comparable to that of the ambient medium. This close relationship between the ambient ISM field and the internal bubble field is ultimately caused by the interaction of the shock wave associated with the expanding bubble with the preexisting turbulent and magnetized ambient ISM. The shock wave propagating ahead of the bubble increases the level of vorticity in the post-shock ISM (Larsson \& Lele 2009), which in turn amplifies the magnetic field in the shocked gas. The amplification rate increases as the coherence length decreases. This amplified field then mixes into the bubble though the bubble-ISM contact discontinuity.

As mentioned above, when the cosmic rays in the bubbles are in contact with magnetic field of this magnitude, then the simulated microwave spectrum may be reconciled with the WMAP observations.

Intermittent activity of Sgr $A^{*}$ - second jet within the Fermi bubbles As the coherence length of ambient field is reduced, the magnetic fields inside the bubbles increase in strength. When these fields reach the magnitude required to account for the level of microwave emission at low galactic latitudes, the plasma $\beta$ approaches unity, i.e., the fields become dynamically important in these regions. Consequently, the magnetic pressure forces inside the bubbles act to redistribute the cosmic ray energy density to higher latitudes. At this point, we are again faced with the problem that sufficiently strong magnetic fields and cosmic rays are not in contact with each other. In order to alleviate this problem, we postulated the existence of a second, more recent AGN activity, and experimented with replenishing cosmic rays by a weaker, second pair of jets. These experiments were successful in explaining the microwave intensity profiles of the WMAP haze while remaining fully consistent with the spatial flatness of the $\gamma$-ray emission from the Fermi bubbles (see Figure 2). Based on the observational evidence, Su \& Finkbeiner (2012) showed that a better match to the $\gamma$-ray morphology of the bubbles can indeed be obtained if an additional pair of jets, anti-symmetric with respect to the disk plane, is 

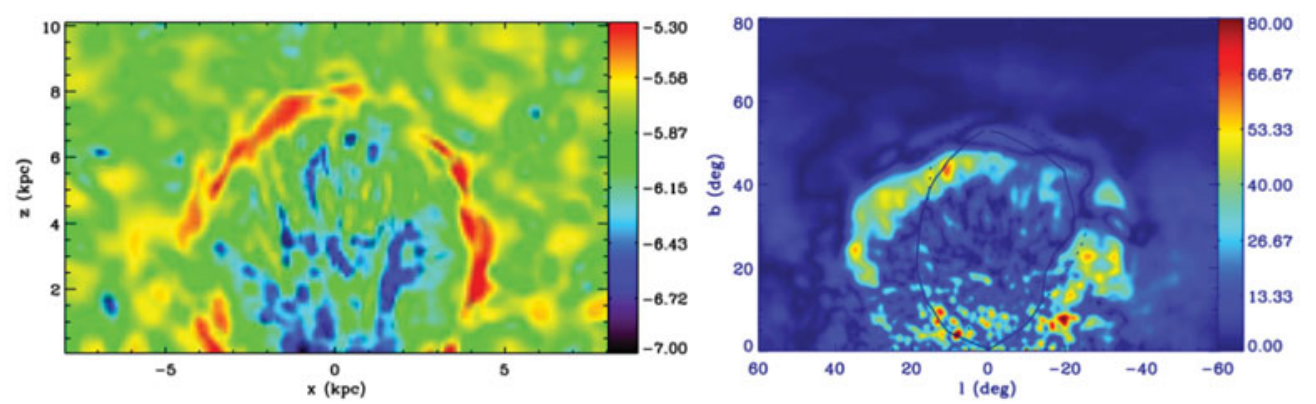

Figure 3. Slice through magnetic field strength distribution (left) and rotation measure

(right). [A COLOR VERSION IS AVAILABLE ONLINE.]

included in the fitting procedure (see also invited talk by Finkbeiner in these proceedings). The Fermi bubbles, including such second pair of internal jets, bear an interesting morphological similarity to Cen A, though this object hosts a substantially more massive central black hole than our Galaxy. Interestingly, Li et al. (2013) recently presented evidence for a sub-parsec X-ray jet in the Galactic center (see Li's poster contribution in these proceedings). This cumulative evidence strongly suggests that the black hole in Sgr A* experienced episodes of past activity.

Radio polarization and rotation measure Our model naturally lends itself to predicting radio signatures of the Fermi bubbles. The relatively high level of field ordering inside the bubbles that was mentioned above leads to high intrinsic linear polarization fractions. The bubble fields tend to be stretched along the jet propagation direction (see radial enhancements in the magnetic field in Figure 3). This is consistent with the linearly polarized intensity S-PASS maps (Carretti et al. 2013) that show high level of polarized emission in features approximately aligned with the jet propagation direction. The polarization fractions predicted by the model are somewhat above those observed, but this discrepancy could easily be resolved by foreground depolarization. We also make predictions for the rotation measure distribution (see Figure 3). Our simulations suggest that the rotation measure could be boosted around the bubbles. This enhancement is especially likely near the bubble-ISM interface but not necessarily perfectly coincident with it. While the likely presence of magnetic field fluctuations unaccounted for in our simulations precludes making any definite predictions as to the level and precise location of these enhancements, this effect could be searched for in latitude-binned rotation measure observations.

We would like to thank the organizers for an exciting and inspiring meeting, and Roland Crocker, Doug Finkbeiner, Jerry Ostriker, Ann Mao, Meng Su, Fred Baganoff, Roman Shcherbakov, and Zhiyuan Li for very stimulating discussions during the workshop. We acknowledge the support from NSF AST 1008454 (PI Ruszkowski), NASA 12-ATP120017 (PI Ruszkowski), and Fermi GSFC 61252, NSPIRES 12-FERMI12-0012 (PI Yang).

\section{References}

Carretti, E., Crocker, R. M., Staveley-Smith, L., et al. 2013, Nature 493, 66

Crocker, R. M. \& Aharonian, F. 2011, Phys. Rev. Lett. 106, 101102

Finkbeiner, D. P. 2004, ApJ 614, 186

Larsson, J. \& Lele, S. K. 2009, Physics of Fluids 21, 126101

Li, Z., Morris, M. R., \& Baganoff, F. K. 2013, arXiv: 1310.0146 
Planck Collaboration 2013, A\&A 554, A139

Su, M., Slatyer, T. R., \& Finkbeiner, D. P. 2010, ApJ 724, 1044

Su, M. \& Finkbeiner, D. P. 2012, ApJ 753, 61

Strong, A. W., et al. 2007, Annual Review of Nuclear and Particle Science 57, 285

Yang, H.-Y. K., Ruszkowski, M., \& Zweibel, E. 2013, MNRAS 2432, arXiv:1307.3551

Yang, H.-Y. K., Ruszkowski, M., Ricker, P. M., Zweibel, E., \& Lee, D. 2012, ApJ 761, 185 